\title{
CRISPR/CAS9-edited pigs as a model for translational research: an update review
}

\author{
N.H. Tinh ${ }^{1}$, A.P.N. Bui ${ }^{2 *}$
}

${ }^{1}$ Institute of Animal Sciences for Southern Vietnam, Hiep Thang quarter, Binh Thang ward, DiAn town, Binh Duong city, ${ }^{2}$ Faculty of Biotechnology, Ho Chi Minh city Open University, 35 Ho Hao Hon street, Ho Chi Minh city, Vietnam

*Corresponding author: Tel: 0084-8-39300086, Email: buiphunamanh@yahoo.com

Journal of Livestock Science (ISSN online 2277-6214) 11: 149-154

Received on 15/7/20; Accepted on 25/9/2020

doi. 10.33259/JLivestSci.2020.149-154

\begin{abstract}
Because of similarities in the anatomy and pathophysiology, pigs have been widely used as a suitable model in biomedical research. As a result, genome edited pigs that mirror the human diseases are of great importance in clinical trials. The advent of CRISPR/Cas9 system has enabled a faster, cheaper and more efficient ways to produce pigs as models in human diseases compared to other methods. In this review, we present an update on application of CRISPR/Cas9 system in creating genetically engineered pigs in translational research.
\end{abstract}

Keywords: CRISPR/Cas9; genome editing; TALEN; translational research; ZFN. 


\section{Introduction}

Translational research in medicine pursues the understanding of disease mechanism and developing new therapies for clinical applications (Bassols et al. 2014; Dmochewitz and Wolf 2015). However, because there are no in vitro systems that can reflect the human whole-body pathophysiology (Perleberg et al. 2018), bridging the "bench to bedside" pathway is often a laborious, inefficient, and costly process. As a result, animal models are often utilized in translational research. Choosing the appropriate animal models that can replicate as closely as possible the human pathology and predict the efficacy and possible side-effects of new treatments are of crucial importance (Aigner et al. 2010).

Traditional biomedical models including rodents, fruit flies, nematode and zebra fish are known for their easy management, sequenced genomes availability (Skarnes et al. 2011). Studies performed in these animals have added a significant amount of valuable knowledge on the molecular basis of human diseases and directions for new therapies in treatments. Nevertheless, there has been a growing concern of the limitations of some animal models because these models do not always accurately represent the human disease mechanism (Klymiuk et al. 2016). Thus, choosing the appropriate animal that can faithfully mirror the human system is in urgent need for biomedical research.

Pigs are highly favored for biomedical studies because of their diversity, ease in breeding and reproduction, large progenies ( 10 piglets per litter), and quick maturation (6 7 months). Moreover, owing to their key similarities with human in anatomy, physiology, disease progression, pigs have been established as a suitable animal models in translational research (Kobayashi, et al. 2012; Bassols, et al. 2014), such as cardiovascular disease, atherosclerosis, cutaneous pharmacology, diabetes, wound repair, ophthalmological studies, and xenotransplantation (Whyte and Prather 2011). With the availability of the swine genome and their genomic similarities to human, generation of genome-edited pigs that tailored for studying mechanism and evaluating therapies for human disease treatments can be rapidly facilitated.

\section{CRISPR/Cas9 system and its mechanism}

The latest genome editing techniques clustered regularly interspaced short palindromic repeats (CRISPR)/ CRISPR-associated protein 9 (Cas9) (CRISPR/Cas9) represents the most advanced platform to create genetically edited pigs for translational research (Proudfoot, et al. 2019). CRISPR/Cas9 was originally discovered in bacteria as a part of adaptive immune system to defend against exogenous nucleic acid invasion (Chylinski et al. 2013). There are three stages in CRISPR-Cas9-mediated defense including adaptation, expression and interference. In the adaptation stage, the foreign DNA fragments, or protospacers, will be acquired and captured into CRISPR arrays. This new spacer acquisition into immune memory system will provide sequence-specific correspondence against plasmids or viruses. In the expression stage, target-specific CRISPR RNA (crRNA) and target-independent transactivating crRNA (tracrRNA) are transcribed from the CRISPR locus. In the interference stage, crRNA and tracrRNA will bind to Cas9 endonuclease protein to target and degrade double strand DNA, thus halt the propagation of exogenous DNA (Huang, et al. 2017; Tait-Burkard, et al. 2018; McFarlane, et al. 2019; Wu and Bazer 2019).

This complex system has been optimized and refined into elegant genome editing system by the integration between crRNA and tracrRNA to become the programmable small guide RNA (sgRNA). In accompany with Cas9 nuclease, sgRNA will target any DNA regions of interest, thus introduce the double strand DNA breaks (DSBs). The DSBs are often repaired by either non homologous end joining (NHEJ) pathway or homologous recombination (HR) pathway. In mammalian cells, the error prone NHEJ pathway is preferred, thereby creating insertions and deletions (indels) mutations (Fig 1).

\section{CRISPR/Cas9 edited pigs in translational research}

CRISPR/Cas9 system has been widely utilized in creating genome-edited pigs as human disease models. Porcine genome modification allows these animals exactly mimic the causative mutations in human genetic disorders, thus more accurately reflect the human diseases phenotypes. As a result, therapies can be applied to test the efficacy of treatments in modelled pigs before proceeding to human clinic trials. A full description of application of CRISPR/Cas9 edited pigs in translational research is presented in Table 1. In this paper, we will highlight some representative and important reports.

\section{Modeling neurodegenerative diseases}

Huntington's disease (HD) and Parkinson's disease (PD) are two most common form of neurodegenerative diseases. With CRISPR/Cas9 system, huntingtin gene-disrupted pigs were created in 2015, indicating similar HDrelated symptoms in human including deficient motor function and respiratory difficulty. In generation of genome 
edited pigs that reflect PD symptoms, two genes Pink1 and Parkin were targeted using CRISPR/Cas9 in a one-step transfection and cloning (Zhou et al. 2015; Yan et al. 2018). These results suggested that genetically modified pigs can be used in establishing neurodegenerative diseases for clinical research.

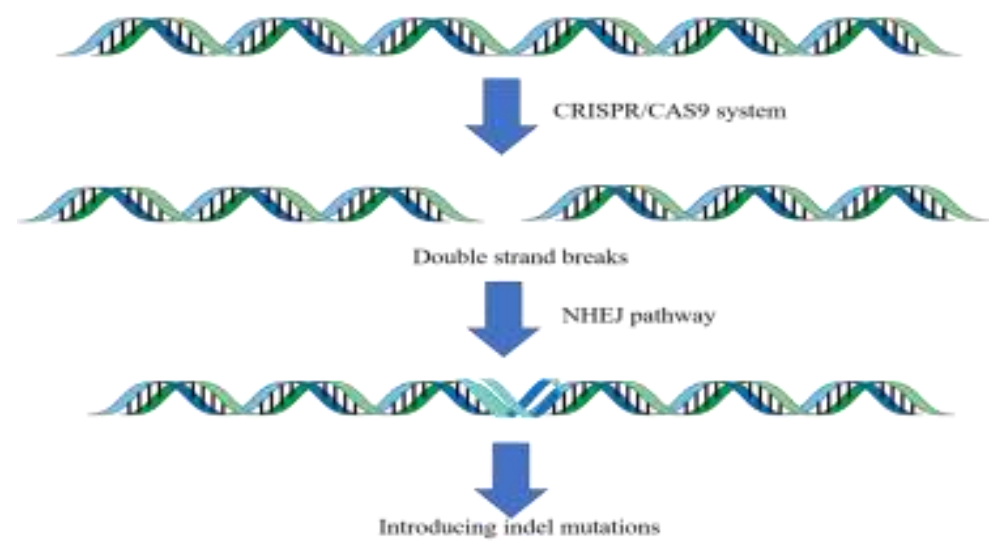

Fig 1. CRISPR/Cas9 working principles

2. Modeling cardiovascular diseases

Statistically, cardiovascular diseases are known to cause the most deaths and disability globally. Functional disruption of low-density lipoprotein receptor (LDLR) and Apolipoprotein E (ApoE) has indicated the development of atherosclerosis, the primary culprit for cardiovascular diseases. In 2017, Huang et al employed CRISPR/Cas9 system to generate ApoE/LDLR knock out pigs. These pigs demonstrated irregular lipid metabolism which is associated to atherosclerosis (Huang et al. 2017).

\section{Modeling cancer}

Acquired mutations that causes uncontrolled development of the cells are the most common source of cancer. By applying CRISPR/Cas9 system, Wang et al (2017) established the genome edited pigs that displayed lung cancer by simultaneously targeting six genes including five tumor suppressor genes TP53, PTEN, APC, BRAC1 and BRAC2, as well as one oncogene KRAS. The CRISPR/Cas9-mediated pigs exhibited signs of pneumonopathy, and human adenocarcinoma pathological feature in the lungs (Wang et al. 2017). Another report by Shen et al (2017) targeted Runt-related transcription factor 3 (RUNX3), which functions as tumor suppressor protein. The results showed that RUNX3 knocked down pigs illustrated carcinogenic phenotypes.

\section{Modeling immunodeficiency}

Severe combined immune deficiency (SCID) is characterized by defective antibody response involved in B lymphocytes recruitment. It has been documented that the development and differentiation of B lymphocytes are crucially regulated by IgM heavy chain. In 2015, by employing CRISPR/Cas9 system, Chen et al has targeted the IgM heavy chain gene to create lymphocytes-deficient pigs (Chen et al. 2015). These genome edited pigs demonstrated the reduction in B lymphocytes and antibody and thus can be further utilized in modeling SCID in clinical trial.

\section{Xenotransplantation}

Pig is widely regarded as suitable organ donor for human transplantation due to the similarity in organ sizes. However, high immune incompatibility often causes the rejection of pig organs by human recipients. Specifically, the presence of Galactose $\alpha(1-3)$ Galactose $(\mathrm{Gal} \alpha(1-3) \mathrm{Gal})$ antigen on the surface of porcine endothelial cells trigger the human immune system to reject the xenograft. To address this hurdle, the enzyme a-1,3galactosyltransferase (GGTA1), which catalyzes the biosynthesis of Gal $\alpha(1-3) \mathrm{Gal}$, was successfully targeted by CRISPR/Cas9 system (Sato, et al. 2014). This result was confirmed by another study led by Petersen et al (2016), which concluded that CRIPSR/Cas9 is a promising tool to pave the way for pig to human xenotransplantation.

Another concern in pig organs donation is the transmission of dormant porcine endogenous retroviruses (PERVs) whose genome is integrated in the porcine genome. PERVs can be activated under certain environmental conditions, thus become infectious. Yang et al (2015) employed CRISR/Cas9 to disrupt all of 62 copies of the PERV pol gene in the immortal pig cell line. Another study led by Niu et al (2017) also deactivated 25 copies of functional PERVs. 
Conclusively, the powerful CRISPR/Cas9 tool can be used in generating successful genome-edited pigs for xenotransplantation.

Table 1. Application of CRISPR/Cas9 edited pigs in translational research

\begin{tabular}{|c|c|c|}
\hline Gene & Function & Source \\
\hline GGTA1 & $\begin{array}{l}\text { Catalyze the synthesis of Galactose } \alpha \text { (1- } \\
\text { 3) Galactose }\end{array}$ & $\begin{array}{l}\text { Sato et al. 2014; } \\
\text { Petersen et al. } 2016\end{array}$ \\
\hline MHC class I & Immune response & Reyes et al. 2014 \\
\hline PERV Pol & Replication & Yang et al. 2015; Niu et al. 2017 \\
\hline $\begin{array}{l}\text { DAZL } \\
\text { APC }\end{array}$ & $\begin{array}{l}\text { Spermatogenesis } \\
\text { Cell adhension }\end{array}$ & Tan et al. 2013 \\
\hline Albumin & & Feng et al. 2015; Peng et al. 2015 \\
\hline $\begin{array}{l}\text { Pink1 } \\
\text { Parkin }\end{array}$ & $\begin{array}{l}\text { Participates in ubiquitination } \\
\text { Protect cells from stress-induced } \\
\text { mitochondrial dysfunction. }\end{array}$ & Wang et al. 2016 \\
\hline RUNX3 & Transcription factor & Kang et al. 2016 \\
\hline $\mathrm{C} 3$ & & Zhang et al. 2017 \\
\hline HUNTINGTIN & long-term memory storage & Yan et al. 2018 \\
\hline ins & & Cho et al. 2018 \\
\hline ApoE & $\begin{array}{l}\text { Mediates the endocytosis of cholesterol- } \\
\text { rich Low-Density Lipoprotein } \\
\text { Involved in the metabolism of fats }\end{array}$ & Huang et al. 2017 \\
\hline $\begin{array}{l}\text { TP53, } \\
\text { PTEN, } \\
\text { APC, } \\
\text { BRAC1 } \\
\text { BRAC2 } \\
\text { KRAS }\end{array}$ & Cancer gene & Wang et al. 2017 \\
\hline $\begin{array}{l}\text { IgM heavy } \\
\text { chain gene }\end{array}$ & 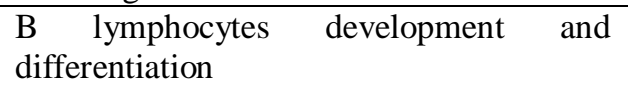 & Chen et al. 2015 \\
\hline PDX1 & Pancreatic development & Wu et al. 2017 \\
\hline
\end{tabular}

Conclusion - Historically, pigs have been established as one of the main meat suppliers for human consumption. Due to the similarities in anatomy, pathophysiology, pigs have been utilized in modelling human diseases in clinical trial. Thanks to the latest genome editing CRISPR/Cas9 system, generation of pigs in translational research has become realistic. Recent reports in clinical trials have demonstrated the feasibility and suitability of genome-edited pigs in studying and identifying treatments in human diseases.

\section{References}

1) Aigner B, Renner S, Kessler B, Klymiuk N, Kurome M, Wünsch A, Wolf E. 2010. Transgenic pigs as models for translational biomedical research. Journal of Molecular Medicine 88:653-664.

2) Bassols A, Costa C, Eckersall PD, Osada J, Sabrià J, Tibau J. 2014. The pig as an animal model for human pathologies: A proteomics perspective. PROTEOMICS - Clinical Applications 8:715-731.

3) Chen F, Wang Y, Yuan Y, Zhang W, Ren Z, Jin Y, Liu X, Xiong Q, Chen Q, Zhang M, Li X, Zhao L, Li Z, Wu Z, Zhang Y, Hu F, Huang J, Li R, Dai Y. 2015. Generation of B Cell-Deficient Pigs by Highly Efficient CRISPR/Cas9-Mediated Gene Targeting. Journal of Genetics and Genomics 42:437-444.

4) Cho B, Kim SJ, Lee E-J, Ahn SM, Lee JS, Ji D-Y, Lee K, Kang J-T. 2018. Generation of insulin-deficient piglets by disrupting INS gene using CRISPR/Cas9 system. Transgenic Research 27:289-300.

5) Chylinski K, Le Rhun A, Charpentier E. 2013. The tracrRNA and Cas9 families of type II CRISPR-Cas immunity systems. RNA biology 10:726-737.

6) Dmochewitz M, Wolf E. 2015. Genetic engineering of pigs for the creation of translational models of human pathologies. Animal Frontiers 5:50-56. 
7) Feng W, Dai Y, Mou L, Cooper DKC, Shi D, Cai Z. 2015. The potential of the combination of CRISPR/Cas9 and pluripotent stem cells to provide human organs from chimaeric pigs. International journal of molecular sciences 16:6545-6556.

8) Huang L, Hua Z, Xiao H, Cheng Y, Xu K, Gao Q, Xia Y, Liu Y, Zhang X, Zheng X, Mu Y, Li K. 2017. CRISPR/Cas9-mediated ApoE-/- and LDLR-/- double gene knockout in pigs elevates serum LDL-C and TC levels. Oncotarget 8:37751-37760.

9) Kang J-T, Ryu J, Cho B, Lee E-J, Yun Y-J, Ahn S, Lee J, Ji D-Y, Lee K, Park K-W. 2016. Generation of RUNX3 knockout pigs using CRISPR/Cas9-mediated gene targeting. Reproduction in Domestic Animals 51:970-978.

10) Klymiuk N, Seeliger F, Bohlooly-Y M, Blutke A, Rudmann DG, Wolf E. 2016. Tailored Pig Models for Preclinical Efficacy and Safety Testing of Targeted Therapies. Toxicologic Pathology 44:346-357.

11) Kobayashi E, Hishikawa S, Teratani T, Lefor AT. 2012. The pig as a model for translational research: overview of porcine animal models at Jichi Medical University. Transplantation Research 1:8.

12) McFarlane GR, Salvesen HA, Sternberg A, Lillico SG. 2019. On-Farm Livestock Genome Editing Using Cutting Edge Reproductive Technologies. Frontiers in Sustainable Food Systems 3.

13) Niu D, Wei H-J, Lin L, George H, Wang T, Lee I-H, Zhao H-Y, Wang Y, Kan Y, Shrock E, Lesha E, Wang G, Luo Y, Qing Y, Jiao D, Zhao H, Zhou X, Wang S, Wei H, Güell M, Church GM, Yang L. 2017. Inactivation of porcine endogenous retrovirus in pigs using CRISPR-Cas9. Science 357:1303-1307.

14) Peng J, Wang Y, Jiang J, Zhou X, Song L, Wang L, Ding C, Qin J, Liu L, Wang W, Liu J, Huang X, Wei H, Zhang P. 2015. Production of Human Albumin in Pigs Through CRISPR/Cas9-Mediated Knockin of Human cDNA into Swine Albumin Locus in the Zygotes. Scientific Reports 5:16705.

15) Perleberg C, Kind A, Schnieke A. 2018. Genetically engineered pigs as models for human disease. Disease models \& mechanisms 11:dmm030783.

16) Petersen B, Frenzel A, Lucas-Hahn A, Herrmann D, Hassel P, Klein S, Ziegler M, Hadeler K-G, Niemann H. 2016. Efficient production of biallelic GGTA1 knockout pigs by cytoplasmic microinjection of CRISPR/Cas9 into zygotes. Xenotransplantation 23:338-346.

17) Proudfoot C, Lillico S, Tait-Burkard C. 2019. Genome editing for disease resistance in pigs and chickens. Animal Frontiers 9:6-12.

18) Reyes LM, Estrada JL, Wang ZY, Blosser RJ, Smith RF, Sidner RA, Paris LL, Blankenship RL, Ray CN, Miner AC, Tector M, Tector AJ. 2014. Creating class I MHC-null pigs using guide RNA and the Cas9 endonuclease. Journal of immunology (Baltimore, Md. : 1950) 193:5751-5757.

19) Sato M, Miyoshi K, Nagao Y, Nishi Y, Ohtsuka M, Nakamura S, Sakurai T, Watanabe S. 2014. The combinational use of CRISPR/Cas9-based gene editing and targeted toxin technology enables efficient biallelic knockout of the $\alpha-1,3$-galactosyltransferase gene in porcine embryonic fibroblasts. Xenotransplantation 21:291-300.

20) Skarnes WC, Rosen B, West AP, Koutsourakis M, Bushell W, Iyer V, Mujica AO, Thomas M, Harrow J, Cox T, Jackson D, Severin J, Biggs P, Fu J, Nefedov M, de Jong PJ, Stewart AF, Bradley A. 2011. A conditional knockout resource for the genome-wide study of mouse gene function. Nature 474:337-342.

21) Tait-Burkard C, Doeschl-Wilson A, McGrew MJ, Archibald AL, Sang HM, Houston RD, Whitelaw CB, Watson M. 2018. Livestock 2.0 - genome editing for fitter, healthier, and more productive farmed animals. Genome Biology 19:204.

22) Tan W, Carlson DF, Lancto CA, Garbe JR, Webster DA, Hackett PB, Fahrenkrug SC. 2013. Efficient nonmeiotic allele introgression in livestock using custom endonucleases. Proceedings of the National Academy of Sciences of the United States of America 110:16526-16531.

23) Wang K, Jin Q, Ruan D, Yang Y, Liu Q, Wu H, Zhou Z, Ouyang Z, Liu Z, Zhao Y, Zhao B, Zhang Q, Peng J, Lai C, Fan N, Liang Y, Lan T, Li N, Wang X, Wang X, Fan Y, Doevendans PA, Sluijter JPG, Liu P, Li X, Lai L. 2017. Cre-dependent Cas9-expressing pigs enable efficient in vivo genome editing. Genome research 27:2061-2071.

24) Wang X, Cao C, Huang J, Yao J, Hai T, Zheng Q, Wang X, Zhang H, Qin G, Cheng J, Wang Y, Yuan Z, Zhou Q, Wang H, Zhao J. 2016. One-step generation of triple gene-targeted pigs using CRISPR/Cas9 system. Scientific Reports 6:20620.

25) Whyte JJ, Prather RS. 2011. Genetic modifications of pigs for medicine and agriculture. Molecular reproduction and development 78:879-891.

26) Wu G, Bazer FW. 2019. Application of new biotechnologies for improvements in swine nutrition and pork production. Journal of animal science and biotechnology 10:28.

27) Wu J, Vilarino M, Suzuki K, Okamura D, Bogliotti YS, Park I, Rowe J, McNabb B, Ross PJ, Belmonte JCI. 2017. CRISPR-Cas9 mediated one-step disabling of pancreatogenesis in pigs. Scientific Reports 7:10487. 
28) Yan S, Tu Z, Liu Z, Fan N, Yang H, Yang S, Yang W, Zhao Y, Ouyang Z, Lai C, Yang H, Li L, Liu Q, Shi H, Xu G, Zhao H, Wei H, Pei Z, Li S, Lai L, Li X-J. 2018. A Huntingtin Knockin Pig Model Recapitulates Features of Selective Neurodegeneration in Huntington's Disease. Cell 173:989-1002.e1013.

29) Yang L, Güell M, Niu D, George H, Lesha E, Grishin D, Aach J, Shrock E, Xu W, Poci J, Cortazio R, Wilkinson RA, Fishman JA, Church G. 2015. Genome-wide inactivation of porcine endogenous retroviruses (PERVs). Science 350:1101-1104.

30) Zhang W, Wang G, Wang Y, Jin Y, Zhao L, Xiong Q, Zhang L, Mou L, Li R, Yang H, Dai Y. 2017. Generation of complement protein C3 deficient pigs by CRISPR/Cas9-mediated gene targeting. Scientific Reports 7:5009.

31) Zhou X, Xin J, Fan N, Zou Q, Huang J, Ouyang Z, Zhao Y, Zhao B, Liu Z, Lai S, Yi X, Guo L, Esteban MA, Zeng Y, Yang H, Lai L. 2015. Generation of CRISPR/Cas9-mediated gene-targeted pigs via somatic cell nuclear transfer. Cellular and Molecular Life Sciences 72:1175-1184. 